\title{
Optical Wireless based on High Brightness Visible LEDs
}

\author{
Grantham Pang, Thomas Kwan, Hugh Liu, Chi-Ho Chan \\ Dept. of Electrical and Electronic Engineering \\ The University of Hong Kong \\ Pokfulam Road \\ Hong Kong \\ Fax: (852)-2559-8738 \\ Tel: (852)-2857-8492 \\ Email: gpang@eee.hku.hk
}

\begin{abstract}
This paper is on a novel use of lighting or signaling devices constructed by light-emitting diodes (LEDs). The idea is on their concurrent use for simultaneous signaling and communications. The visible light from LEDs can be modulated and encoded with audio or data signal. Hence, an LED indicator lamp or traffic light can become an information broadcasting device. Such a system made up of high brightness visible LEDs can provide the function of open space, wireless broadcasting of audio or data signal. An implemented system with experimental results is shown. A portable receiver is designed to demodulate the optically transmitted information. The distance for audio transmission of an LED traffic light can reach over $20 \mathrm{~m}$. The optical link employs intensity modulation with direct detection. The system is limited to line-of-sight communications. However, this concurrent use of LEDs will open up many new applications.
\end{abstract}

\section{INTRODUCTION}

The superiority of light-emitting diodes (LEDs) over incandescent lights is well-supported. This is due to their long life expectancy, high tolerance to humidity, low power consumption and minimal heat generation. Light-emitting diodes (LEDs) are being used in many message display boards, signal devices, or any other means of illumination. One important characteristic of LEDs is that they are semiconductor devices and are capable of fast switching with the addition of appropriate electronics. That is, the visible light emitted by the LEDs can be modulated and encoded with audio information for broadcasting. Therefore, in addition to their normal function of being an indication and illumination device, LEDs can be used as a communication device for the transmission and broadcasting of audio information. Hence, it becomes part of a wireless optical communication system. This paper describes an audio information system for the broadcasting of audio signal using visible light emitting diodes.

Nowadays, billions of visible LEDs are produced each year and the emergence of high brightness AlGaAs and AlInGaP devices has given rise to many new markets $[1,2,3]$. The surprising growth of activity in relatively old topic of LEDs is spurred by introduction of AllnGaP devices. Recently developed AlGaInN materials have led to improvements in the performance of bluish-green LEDs [1]. The entire visible spectrum is occupied with these LEDs which have luminous efficacy peaks much higher than those for incandescent lamps $[3,4]$. This advancement has led to the production of largearea full-color LED displays for outdoors with diverse industrial applications.

The novel idea of this paper is to modulate the light waves from the visible LEDs for communication purpose [5]. This concurrent use of visible LEDs for simultaneous signaling and communication leads to many new and interesting applications. The work is based on the idea of fast switching of LEDs, and the modulation of the visible light waves for free space communications. The feasibility of such an approach has been examined and hardware has been implemented with experimental results. An implementation of an optical link has been carried out using an LED traffic signal head as a transmitter. The LED traffic light can be used for either audio or data transmission. Audio messages can be sent using the LED transmitter and a receiver located at a distance around $20 \mathrm{~m}$ away can play back the messages with a speaker. For data transmission, digital data can be sent using the same LED transmitter, and experiments have been setup to send a speed limit or location ID information.

The work reported in this paper differs from the use of infrared (IR) radiation as a medium for short-range wireless communications [6,7]. Currently, infrared links and localarea networks are available. IR transceivers for use as IR data links are widely available in the market at a very low cost. Some systems are comprised of infrared transmitters which convey speech messages to small receivers carried by persons with severe visual impairments. The Talking Signs system is one such infrared remote signage system developed at the Smith-Kettlewell Rehabilitation Engineering Research Center $[8,9]$. It can provide a repeating, directionally selective voice message which originates at a sign. Comparison between the infrared and other media such as radio and microwave is given in [10]. Anyhow, there has been very little work on the use of visible light as a communication medium. The availability of high brightness LEDs also makes the visible light medium even more feasible. All products with visible LED components (like LED traffic signal head) can be turned into an information beacon.

It has long been realized that visible light has the potential to be modulated and used as a communication channel with 
entropy. The application has to make use of the directional nature of the communication medium because the receiver requires a line-of-sight to the audio system or transmitter. The locations of the audio signal broadcasting system and the receiver are relatively stationary. Since the relative speed between the receiver and the source are much less than speed of light, the dopplar frequency shift observed by the receiver can be safely neglected. It should be noted that the transmitter provides easy targets for the line-of-sight reception by the receiver. This is because the LEDs, being on at all time, are also indicators of the locations of the transmitter. The transmitter can broadcast with a viewing angle close to 180 degrees. This paper aims to present an application of high brightness visible LEDs for establishing an optical free space links. Next, a visible light LED audio information system is described.

\section{SYSTEM DESCRIPTION}

A block diagram representation of the schematic diagram of the transmitter design is shown in Figure 1. The audio signal from the cassette tape or CD player has small amplitude and hence amplification of this audio signal is necessary. The audio amplifier is used to amplify the weak audio signal and to shift the average voltage level of the audio signal to an appropriate level so that the signal is within the capture range of VCO. A VCO chip is used to modulate the incoming audio signal variations from the audio amplifier and generate the FM signal. A square wave VCO is used instead of sine wave because there are only two states (ON and OFF) for the LEDs. The carrier frequency is set at $100 \mathrm{kHz}$ with a maximum frequency deviation of $\pm 50 \mathrm{kHz}$. The modulated signal is transmitted by the switching of the LEDs. The frequency of switching is high enough such that the perceivable light appears to be constantly illuminated to the human eye.

A block diagram schematic of the receiver design is shown in Figure 2. The photo-detector is used to detect light signal from the transmitter and convert the signal into an electrical signal. The limiting pre-amplifier is used to amplify the electrical signal from the photo-detector for the next stage. The data reproducing circuit is used to reconstruct the square wave. The differentiator circuit is used to produce pulses according to the square wave. The pulse generator is used to convert the pulses from the differentiator into sharp pulses for use by the integrator and envelope detector in the next stage for the demodulation of the signal. The band-pass filter is then used to smooth out the distortions from the integrator and envelope detector to produce an appropriate waveform. Finally, the power amplifier is used to amplify the weak signal from the band-pass filter so that the audio signal would be comfortable for hearing. Below is a more detailed description of each stage.

The photo-detector circuit consists of a photodiode and a resistor. One end of the photodiode is coupled to the current limiting resistor with the other end coupled to ground. Since the signal from the photo-detector circuit is small, amplification is needed for the next stage. The limiting pre- amplifier circuit consists of two op-amplifiers as well as some resistors and diodes. The diodes are used to limit the input voltage level to a desired level (such as between $-0.7 \mathrm{~V}$ and $0.7 \mathrm{~V}$ ). This circuit aims to amplify the input signal to a certain level and a comparator is used to produce rectangular signal pulses. Two pre-amplifiers are used in this circuit because using one pre-amplifier will require a very high gain amplifier. Hence, two pre-amplifiers with lower gain each is used to achieve a high gain but with reduced noise.

Next, a data reproducing circuit is used which consists of an operational amplifier, a resistor and two NAND Schmitt triggers. The function is to produce rectangular pulses from the amplified signal in the previous stage. An operational amplifier is used as a comparator with uses virtual ground as a reference. Two NAND Schmitt trigger gates are used to enhance the noise immunity and to correct edges from low to high voltage level due to the slew rate of amplifier. Two NAND Schmitt trigger gates are used instead of one so that the signal will not be inverted. Then, a differentiator circuit consisted of a capacitor and a resistor is used to detect the leading edges of the pulse with the trailing edges blocked by a diode. Next, there is the circuit of a pulse generator. A Schmitt trigger gate is used as a pulse generator and the output gives the inverted version of pulses from the differentiator.

An integrator and envelope detector can be found in the next stage. The integrator is an envelope detector and double integrations are carried out. If the inverted pulses from the pulse generator contain high frequency, the frequency of integration is higher and the voltage level of the output would be higher. However, if the inverted pulses contain low frequency, the frequency of integration is lower and the voltage level of the output would be lower. In this way, the modulated signal would be reconstructed. Next, a band-pass filter is used. The output signal from the previous stage, integrator and envelope detector, has many distortions. A band-pass filter is used to filter out all the high frequency distortions. The higher cut-off frequency depends on a capacitor and resistor. A lower cutoff is also used to filter out the low frequency noise such as the $50 \mathrm{~Hz}$ power line frequency. The output signal from the band-pass filter is an audio signal. The final stage of the receiver circuit is a power amplifier and the output of which is connected to the speaker. The objective is for the delivery of the audible messages through a speaker or head phone/ear jack.

\section{APPLICATIONS OF THE VISIBLE LED SYSTEM}

We shall now discuss potential applications of the above audio information system and companion receiver. One application is for LED traffic lights. Nowadays, high brightness LEDs are increasing being used in traffic lights. This is mainly due to the low power consumption and minimal maintenance required for LED-based traffic lights, which can be translated into considerable cost savings each year [11]. For example, in the City of Philadelphia, U.S.A., the city is replacing all of its 28,000 red signals with LEDs, with an estimated annual cost savings of US 1.2 million. The 
next stage of development will involve the three-color LED signals. With the idea and development described in this paper, an LED traffic light can be used as an audio broadcasting device, in addition to their normal function of being an indication and signaling device. A receiver some distance away pointing at the traffic light can receive voice messages. For drivers, the message can announce the duration for the next signal change. For pedestrians or people with visual impairments, the voice message can tell the location or direction information.

The above development allows a concurrent use of traffic lights because it can broadcast local traffic information, location and road information to both pedestrians and road users, and simultaneously perform its normal function of being a traffic signaling device. The LED traffic light becomes a new kind of short-range information beacon. Essentially, all LED-based traffic signs, displays or illumination devices can perform the above functions.

Other applications can be found indoors in a museum or exhibition hall environment. The information on individual exhibit can be broadcast via a plurality of LEDs, which is also used for the purpose of illumination. With the guest pointing the receiver to the relevant LEDs on a transmitter, with the head phone or ear jack attached to the portable receiver, he can listen to the audio message about the specific exhibit item he is interested. Thus, the indoor environment can remain quiet while the guests stroll in the museum. A major advantage over conventional broadcasting system is that an individual with a receiver has the freedom of choice in receiving specific messages without hearing any unwanted announcement, music or commercials.

\section{IMPLEMENTATION AND EXPERIMENTAL RESULTS}

An LED traffic signal head made up of 441 high brightness LEDs (Figure 3) has been implemented in the Industrial Automation Laboratory at The University of Hong Kong. Each LED is an HP high intensity AlInGaP type with a luminous intensity of $1100 \mathrm{mcd}$ at rated $20 \mathrm{~mA}$ driving current, and the viewing angle is 30 degrees. Figure 4 shows the implemented receiver.

The specifications of the LED traffic signal head is given below:

\begin{tabular}{|l|l|}
\hline Signal color & Red \\
\hline Construction & $\begin{array}{l}441 \text { red ultra-bright LEDs } \\
\text { with a luminous intensity } \\
\text { of 2000 mcd @20mA }\end{array}$ \\
\hline Diameter & 8 inches \\
\hline Nominal usage & $17 \mathrm{~V} ; 2 \mathrm{~A}$ \\
\hline Nominal power consumption & $34 \mathrm{~W}$ \\
\hline Luminous intensity & $300 \mathrm{~cd}$ \\
\hline $\begin{array}{l}\text { Viewing angle 20 1/2(half } \\
\text { power) }\end{array}$ & 30 degrees \\
\hline $\begin{array}{l}\text { Distance for audio transmission } \\
\text { (outdoors) }\end{array}$ & Over $20 \mathrm{~m}$ \\
\hline
\end{tabular}

The radiation pattern of the LED traffic light is given in Figure 5. An HP Audio Analyzer, which has a low distortion signal source with a signal analyzer, is then used for audio measurement of the visible light LED audio broadcasting panel. The frequency response of communication channel occupied by the audio signal was determined. Here, the frequency of audio signal transmitted via the LEDs was varied and the response was observed using the HP audio analyzer. The frequency response is not flat as may be expected from the enormous bandwidth of visible light. This is due to the limitations inherently governed by the VCO in the transmitter and the discriminator used at the receiver. The frequency response characteristic of the system is shown in Figure 6 . In another measurement on the signal-to-noise ratio, the result is shown in Figure 7.

The same LED traffic light has also been used for data transmission. Digital data can be sent using the same LED transmitter, and experiments have been setup to send digital information. A receiver, which resembles a portable traveler location system, has been implemented to obtain the demodulated signal. Figure 8 shows the block diagram of the receiver.

A bit error rate experiment for the LED traffic light has been performed. Figure 9 shows the experimental setup. In the experiment, frames of data were transmitted continuously from a computer to the serial communication interface circuit via the printer port of the computer. The modulated signal is transmitted by the LED traffic light. The visible light signal was transmitted to the receiver and the serial communication interface performs demodulation of the data. The computer at the receiver side would compare the received data with the transmitted data. The number of error bits would be recorded.

The data frames transmitted by the LED traffic light contained pseudo-random series of data divided into 31 data blocks. The transmission speed of the visible light communication channel is $128 \mathrm{kbps}$. The indoor ambient light power was measured by an optical power meter which was found to be $12 \mu \mathrm{W}$. Table 1 shows the results of the bit error rate test.

\begin{tabular}{|l|l|}
\hline $\begin{array}{l}\text { Power of visible light } \\
\text { signal at the receiver }\end{array}$ & Bit Error Rate \\
\hline $0.5 \mu \mathrm{W}$ & $2.1315 \times 10^{-3}$ \\
\hline $0.6 \mu \mathrm{W}$ & $5.2177 \times 10^{-7}$ \\
\hline $0.7 \mu \mathrm{W}$ & $2.4835 \times 10^{-1}$ \\
\hline $0.8 \mu \mathrm{W}$ & $9.1982 \times 10^{-8}$ \\
\hline $1.8 \mu \mathrm{W}$ & $<2.2155 \times 10^{-10}$ \\
\hline
\end{tabular}

Table 1 Bit error rate experiment results

It is observed that the bit error rate decreases as the power (or luminance) transmitted by the LED traffic light increases. In other words, the brighter the traffic light, the smaller is the bit error rate-occurred at the receiver. 
In a real situation, there will be other visible light sources nearby. One example would be the head light of a vehicle traveling in the opposite lane. Thus, the effect of head light was evaluated. This situation was simulated by placing a lamp with $100 \mathrm{~W}$ light bulb besides the LED traffic light. The bit error rates for the traffic light signal at $0.7 \mu \mathrm{W}$ were compared. Without the head light, the bit error rate is 2.4835 $\times 10^{-7}$. With the headlight, the bit error rate is $1.1232 \times 10^{-6}$. This shows that the head light would increase the bit error rate.

\section{DISCUSSIONS \& CONCLUSIONS}

High brightness LEDs are getting more popular and are opening up a number of new applications, especially with improved efficiency and new colors. In this paper, the novel idea based on the fast switching of LEDs and the modulation of the visible light is developed into a new kind of information system. A visible LED audio system makes use of visual light rays to transmit audio messages to a remotely located receiver is described. Such a system made up of high brightness visible LEDs can provide the function of open space, wireless broadcasting of audio signal. It can be used as an information beacon for short distance communication.

As a medium for wireless short-range communication, visible light has both advantages and disadvantages when compared with infrared, microwave and radio media. On one hand, LEDs and photodetectors capable of high-speed operation are available at low cost. Like the infrared, the visible spectral region is unregulated worldwide and FCC licenses are not necessary, as the commission does not regulate the visible light frequencies. Both infrared and visible light penetrate through glass, but not through walls. For the transmission to be possible, there must be no obstructions standing in the way of the visible LED light beam as it requires a clear line of sight (LOS) between the sending side (LED) and receiving side, whereas infrared also allows non-directed and non-LOS link design. Like microwave, visible LED light beam follows a straight-line path and is well suited for the wireless delivery of large quantities of voice, data information. In practical use, one should take advantage of this highly directional feature of LEDs.

On the other hand, LEDs also has many drawbacks. It is suitable only for short-range, as the photodetector current is proportional to the received power. Intensity modulation with direct detection seems the only practical transmission method. The Signal-to-Noise Ratio (SNR) of a directdetection receiver is proportional to the square of the received optical power. It should also be mentioned that the relationship between the radiant intensity and distance from the receiver follows the inverse square law. Hence, as a communication medium, it has limited range and is subject to noise arising from sunlight and incandescent lighting and fluorescent lighting. - - It is not suitable for broadcasting of signal over a wide coverage area and over long ranges.
To conclude, any illumination device made use of high brightness visible LEDs can be used as a kind of short-range information beacon. An interesting example given in this paper is an LED traffic light for the support of roadside-tovehicle communications. There would be many novel use of visible light from LEDs as a communication medium. This concurrent use of LEDs for simultaneous signaling and communications will open up many new applications.

\section{REFERENCES}

[1] G.B. Stringfellow and M.G. Craford, "High Brightness Light Emitting Diodes", Semiconductors and Semimetals Vol. 48, Academic Press, 1997.

[2] M.G. Craford, "LEDs Challenge the Incandescents", IEEE Circuits and Devices, pp. 24-29, September 1992.

[3] K. Werner, "Higher visibility for LEDs", IEEE Spectrum, pp.30-39, July, 1994.

[4] P.P. Smyth, P.L. Eardley, K.T. Dalton, D.R. Wisley, P. McKee and D. Wood, "Optical Wireless - a prognosis", Proc. On Wireless Data Transmission, SPIE Vol. 2601, pp. 212-225, October 23-25, 1995.

[5] G. Pang, E. Yang, C. Yeung, M. Hong, D. Yang, H. Liu, T. Kwan, L. Ko, C. Chan, "Concurrent Display and Data Communication using LEDs", U.S. Patent Application, filed on March 19, 1999.

[6] T.S. Chu and M.J. Gans, "High speed infrared local wireless communication", IEEE Communications Magazine, pp. 4-10, August 1997.

[7] M. Meyer, "Infrared LEDs", Compound Semiconductor, pp. 39-40, May/June, 1996.

[8] W. Loughborough, W. "Talking Signs - An Accessibility Solution for the Blind and Visually Impaired." $12^{\text {th }}$ C.M.B.E.C. / Ist Pan Pacific Symp., Vancouver, Canada, 1986.

[9] B.L. Bentzen, P.A. Mitchell, "Audible Signage as a Wayfinding Aid: Comparison of "Verbal Landmarks®" and "Talking Signs $($ ", Research report for the California Council of the Blind and the Association for the Advancement of the Blind, 1995, http://www.ski.org/rerc/WCrandall/ACBPAPER.HTM.

[10] J.M. Kahn and J.R. Barry, "Wireless Infrared Communications", Proceedings of the IEEE, Vol. 85, No.2, pp.265-298, Feb 1997.

[11] J. O'Connell, "The Philadelphia story", Traffic Technology International, UK \& International Press, pp. 106-110, Aug/Sept. 19.97. 


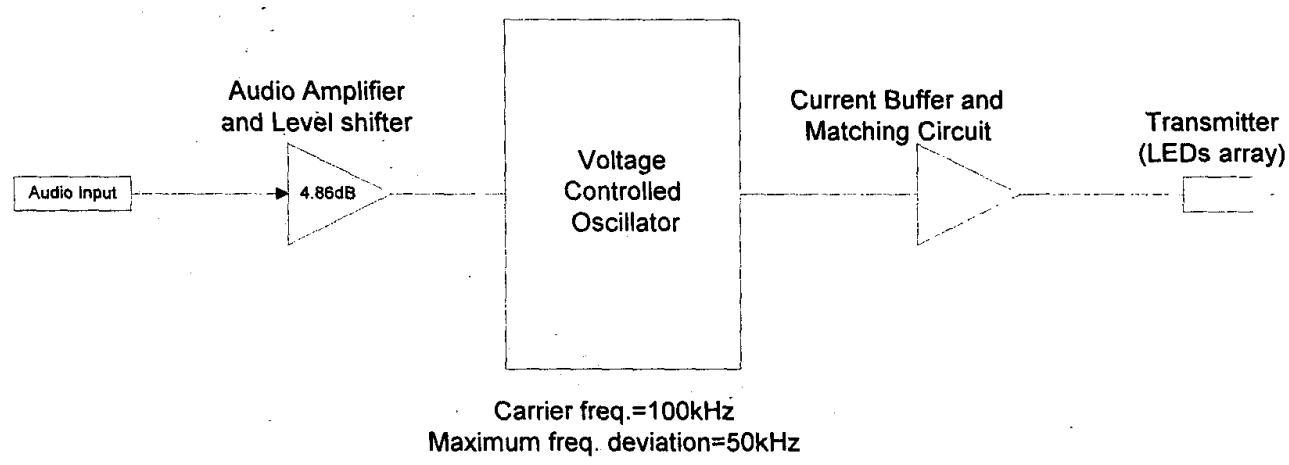

Fig. 1 Block diagram representation of the schematic diagram of the transmitter design.

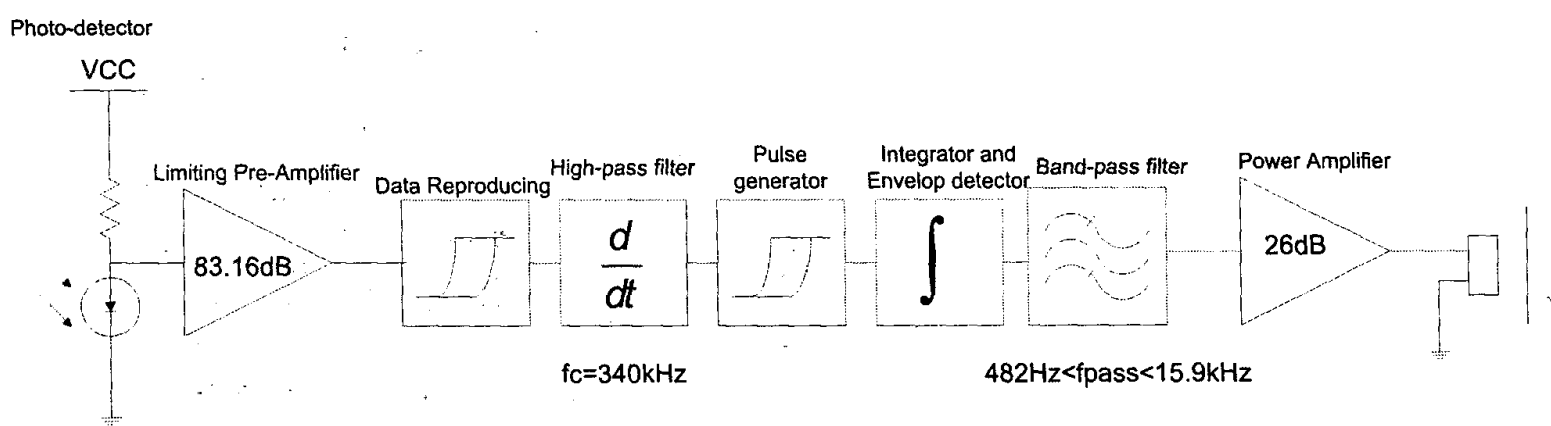

Fig. 2 Block diagram representation of the schematic diagram of the receiver design.
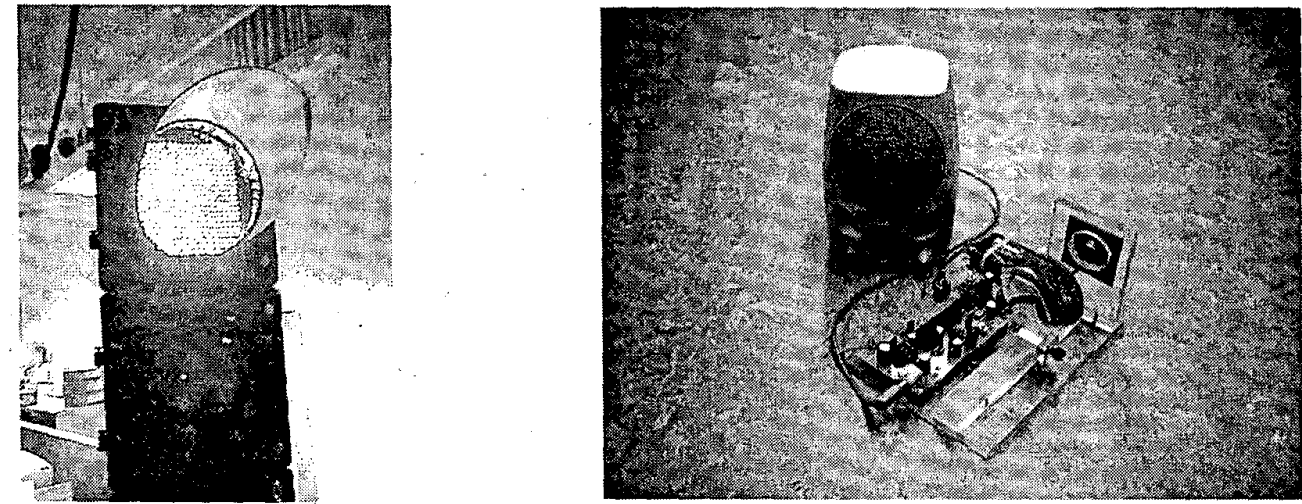

Fig. 3 Photograph of the LED traffic light.

Fig. 4 Photograph of the receiver. 


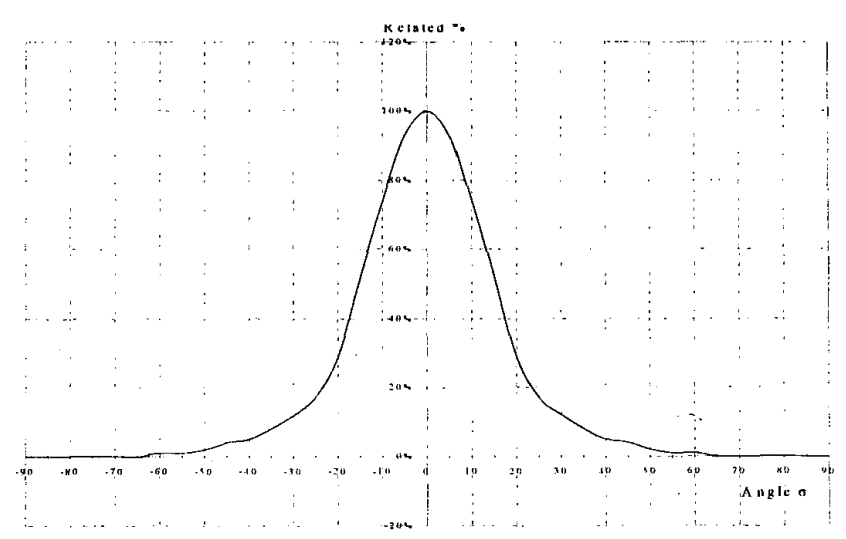

Fig 5. Radiation pattern of the LED traffic light.

Frequency response characteristics
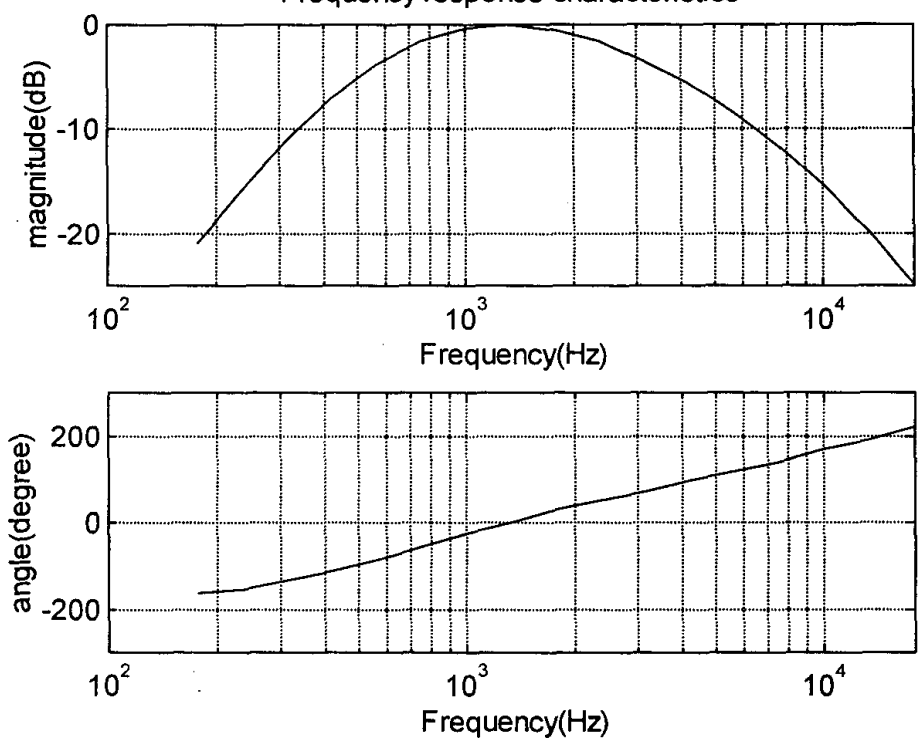

Fig 6. Frequency response characteristics of the system.

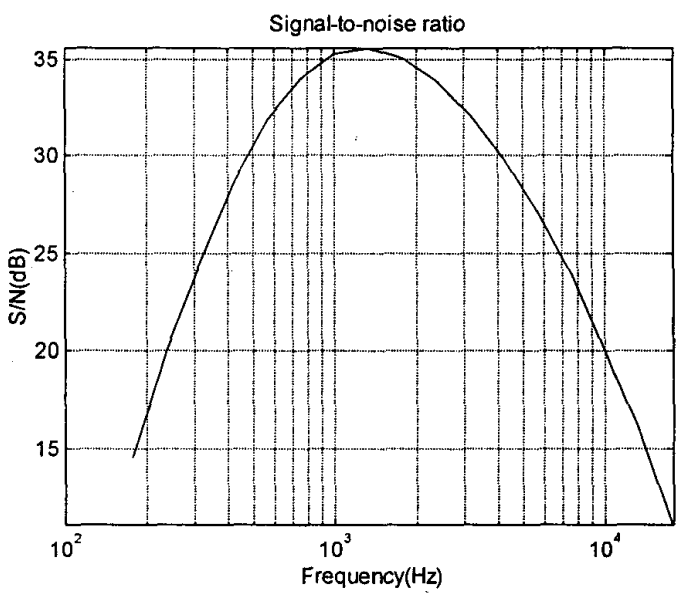

Figure 7. Measurement on the signal-to-noise ratio. 


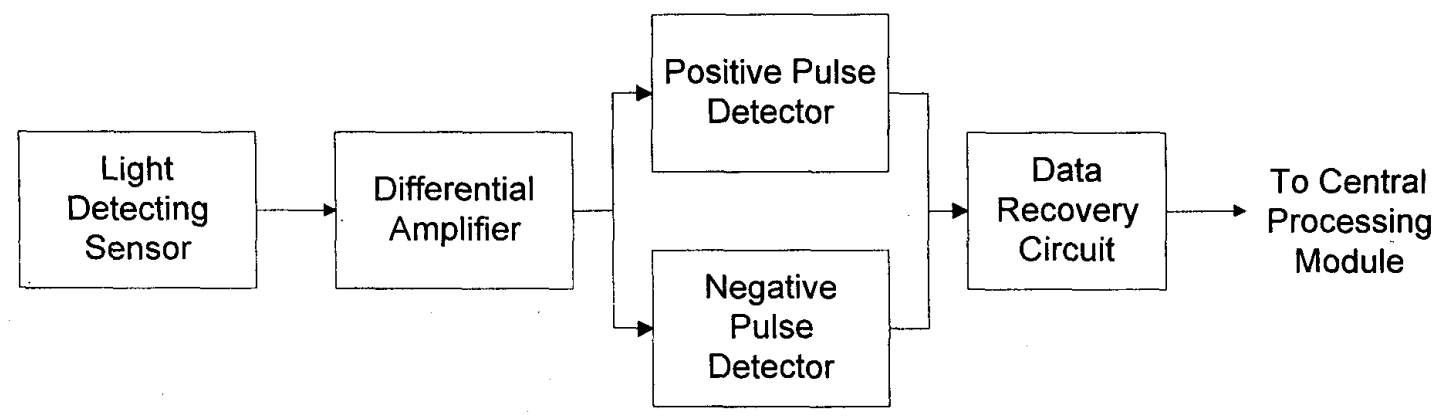

Figure 8. Visible light receiver

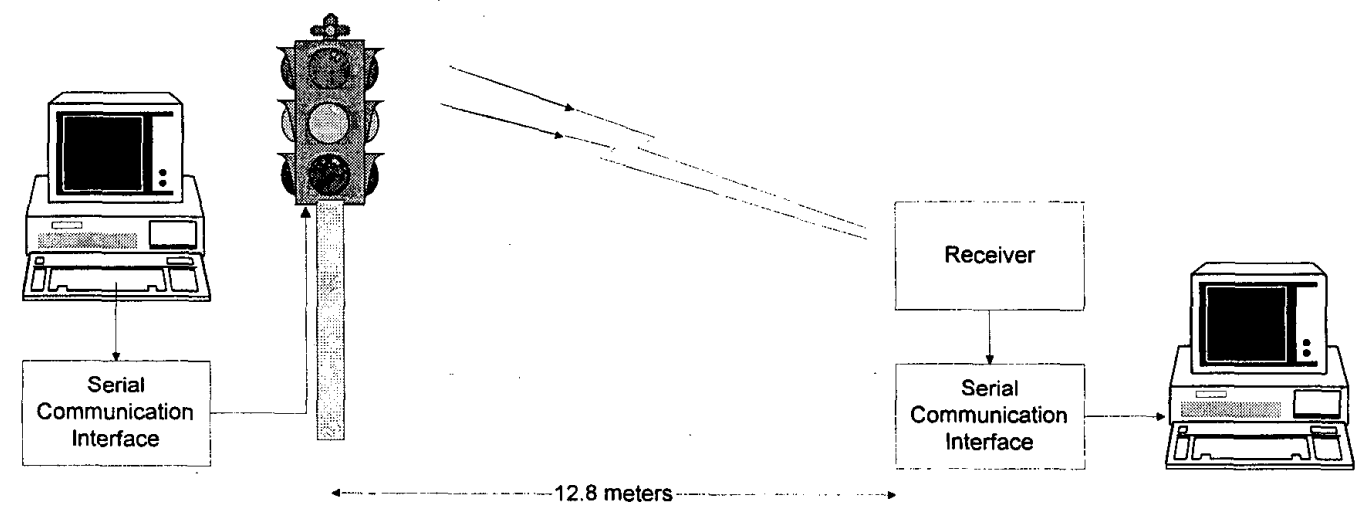

Figure 9. Bit error rate experimental setup 